\title{
Deflection and translation of a ray traversing an optical system, nodal rays and the optical axis
}

\author{
W F Harris*, S D Mathebula ${ }^{\dagger}$ and A Rubin
}

* \$Department of Optometry, University of Johannesburg, P O Box 524, Auckland Park, 2006

South Africa, and Department of Optometry, University of Limpopo, Private Bag x1106, Sovenga, 0727 South Africa

*wharris@uj.c.za, ${ }^{\dagger}$ solanim@ul.ac.za and ${ }^{\dagger}$ arubin@uj.ac.za

Received 9 June 2009; revised version accepted 27 October 2009

\begin{abstract}
Expressions are derived for the deflection and transverse translation of a ray as it traverses an arbitrary optical system. The system may be astigmatic and have relatively decentred refracting elements. The expressions are in terms of the fundamental properties of the system. Because nodal rays are rays that undergo no deflection the results lead immedi-
\end{abstract}

ately to expressions that define nodal rays through the system. An optical axis of an optical system is a nodal ray that is a straight line through the system. This allows one to write an expression that determines the optical axis of an arbitrary optical system.

Key words: deflection, transverse translation, nodal rays, optical axis

\section{Introduction}

A ray entering an optical system usually leaves the system with a different direction and at a different distance from the longitudinal axis. Thus the ray undergoes deflection and transverse translation as it traverses the system. The deflection and transverse translation are shown as the scalars $\Delta a$ and $\Delta y$ in Gaussian optics in Figure 1 and as the vectorial quantities $\Delta \mathbf{a}$ and $\Delta \mathbf{y}$ in linear optics in Figure 2. An expression for the deflection was needed for another study ${ }^{1}$, and deflection and transverse translation provide alternative and relatively simple approaches to finding nodal rays and optical axes which have been topics of recent research ${ }^{2,3}$. Accordingly the purpose of this note is to derive general expressions for the deflection and transverse translation of a ray across an arbitrary optical system and then to show how expressions for nodal rays and the optical axis of the system follow directly.

In order to prepare the groundwork for the general case we first examine deflection and transverse translation in the simpler case of the centred system in Gaussian optics. With the understanding provided by this two-dimensional approach we turn to the general three-dimensional problem of deflection and transverse translation of a ray through a system that may contain astigmatic and relatively decentred elements. The optical model is linear optics. The results are then applied to finding nodal rays and optical axes; for both nodal rays and optical axes there is no deflection and for optical axes the transverse translation is simply related to the inclination of the nodal ray and the length of the system. For the basics of Gaussian and linear optics the reader is referred to the text by Guillemin and Sternberg ${ }^{4}$. For what follows the reader is also referred to two recent papers ${ }^{2,3}$.

*PhD FRSSAf

†Optom

\$DPhil 


\section{Gaussian optics}

Consider an arbitrary optical system $\mathrm{S}$ in Gaussian optics (Figure 1). $\mathrm{Z}$ is a longitudinal axis and $\mathrm{T}_{0}$ and $\mathrm{T}$ are the entrance and exit planes of $\mathrm{S}$. The indices of refraction are $n_{0}$ immediately before the system and $n$ immediately after it. Suppose $Z$ coincides with the optical axis of the system. The optical nature of $\mathrm{S}$ is characterized by the $2 \times 2$ transference

$$
\mathbf{S}:=\left(\begin{array}{ll}
A & B \\
C & D
\end{array}\right) .
$$

$\mathrm{A}$ is the dilation, $\mathrm{B}$ the disjugacy, $\mathrm{C}$ the divergence and $\mathrm{D}$ the divarication of the system; they are four fundamental scalar optical properties of the system. The dioptric power of $\mathrm{S}$ is defined by

$$
F:=-C
$$

It is convenient to develop the analysis in terms of $C$. At any point one may obtain an expression in terms of dioptric power $F$ by replacing $C$ by $-F$.

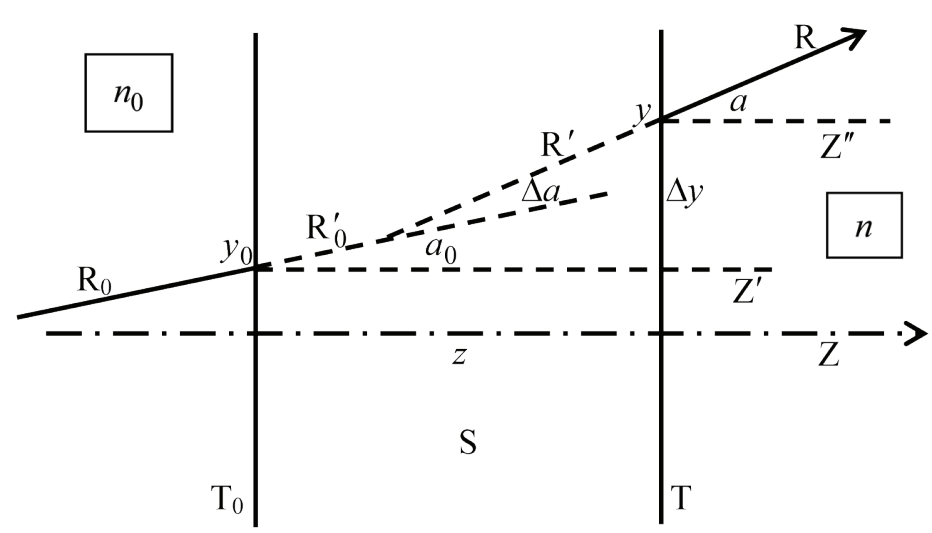

Figure 1: Deflection $\Delta a$ and transverse translation $\Delta y$ of a ray through an arbitrary system $\mathrm{S}$ in Gaussian optics (two-dimensional). $\mathrm{T}_{0}$ and $\mathrm{T}$ are transverse planes a distance $z$ apart and orthogonal to longitudinal axis $\mathrm{Z}$; they are the entrance and exit planes of $S . R_{0}$ is the incident segment of a ray. It intersects $T_{0}$ at $y_{0}$ and has inclination $a_{0} \cdot \mathrm{R}_{0}^{\prime}$ is an extension of $\mathrm{R}_{0}$ and $Z^{\prime}$ and $Z^{\prime \prime}$ are parallel to $Z$. The ray emerges at $T$ at the point $y$ and with inclination $a$. $\mathrm{R}^{\prime}$ is an extension of $\mathrm{R}$. The indices of refraction immediately before and immediately after system $\mathrm{S}$ are $n_{0}$ and $n$ respectively. The deflection is given by Equations 17 and 19 and the transverse translation by Equation 16 and 18.
Now consider a ray through system $\mathrm{S}$. Its incident segment is $\mathrm{R}_{0}$ in Figure 1 and its emergent segment is $\mathrm{R}$. $\quad \mathrm{R}_{0}^{\prime}$ and $\mathrm{R}^{\prime}$ are extensions of $\mathrm{R}_{0}$ and $\mathrm{R}$ respectively. At $\mathrm{T}_{0}$ segment $\mathrm{R}_{0}$ has transverse position $y_{0}$ and inclination $a_{0}$ relative to $\mathrm{Z}$. The reduced inclination is

$\alpha_{0}:=n_{0} a_{0}$

Together $y_{0}$ and $\alpha_{0}$ constitute

$\rho_{0}=\left(\begin{array}{l}y_{0} \\ \alpha_{0}\end{array}\right)$,

the state of the ray at incidence. We shall refer to $\rho_{0}$ as the incidence of the ray.

The ray emerges from $\mathrm{S}$ at $\mathrm{T}$ with transverse position $y$ and inclination $a$. Its reduced inclination is

$\alpha:=n a$.

The state of the ray at emergence is

$\rho=\left(\begin{array}{l}y \\ \alpha\end{array}\right)$,

which we shall refer to simply as the emergence of the ray.

The emergence and incidence of a ray traversing system $\mathrm{S}$ are related by

$\mathrm{S} \rho_{0}=\rho$.

Substituting from Equations 1 and 3 to 6 we obtain the pair of scalar equations

$A y_{0}+n_{0} B a_{0}=y$

and

$C y_{0}+n_{0} D a_{0}=n a$.

Because of symplecticity the inverse of $\mathbf{S}$ exists for all systems. Hence we can rewrite Equation 7 as

$\mathbf{S}^{-1} \boldsymbol{\rho}=\rho_{0}$. 
Now

$\mathbf{S}^{-1}=\left(\begin{array}{cc}D & -B \\ -C & A\end{array}\right)$

Substituting into Equation 10 we obtain

$D y-n B a=y_{0}$

and

$-\mathrm{Cy}+n A a=n_{0} a_{0}$.

We define

$\Delta y:=y-y_{0}$

and

$\Delta a:=a-a_{0}$.

$\Delta y$ is the change in position of the ray from incidence to emergence relative to longitudinal axis Z; it is the transverse translation of the ray. Similarly $\Delta a$ is the change in inclination of the ray across the system; it is the deflection of the ray. $\Delta y$ and $\Delta a$ are shown in Figure 1.

Substituting from Equation 8 into Equation 14 we obtain

$$
\Delta y=(A-1) y_{0}+n_{0} B a_{0} .
$$

Similarly, substitution from Equation 9 into Equation 15 results in

$\Delta a=\mathrm{C} y_{0} / n+\left(n_{0} D / n-1\right) a_{0}$.

From Equations 12 to 15 we obtain the transverse translation and the deflection of a ray in terms of its emergent state,

$\Delta y=(1-D) y+n B a$

and

$\Delta a=\mathrm{C} y / n_{0}+\left(1-n A / n_{0}\right) a$.

Equations 16 and 17 give the transverse translation and the deflection of the ray in terms of the incident state of the ray; Equations 18 and 19 do the same except they are in terms of the emergent state of the ray.

If the indices before and after the system are the same then Equations 17 and 19 reduce to

$\Delta a=\mathrm{C} y_{0} / n_{0}+(D-1) a_{0}$

and

$\Delta a=\mathrm{C} y / n+(1-A) a$.

If the ray is incident parallel to axis $\mathrm{Z}$ then $a_{0}=0$ and Equations 16 and 17 become

$\Delta y=(A-1) y_{0}$

and

$\Delta a=\mathrm{C} y_{0} / n$.

\section{Linear optics}

Above we looked at the simplest case of transverse translation and deflection of a ray in two dimensions across a system centred on its optical axis. We now go to the opposite extreme: transverse translation and deflection across a system that may be astigmatic and with refracting elements not necessarily centred on the longitudinal axis. The situation is represented in Figure 2.

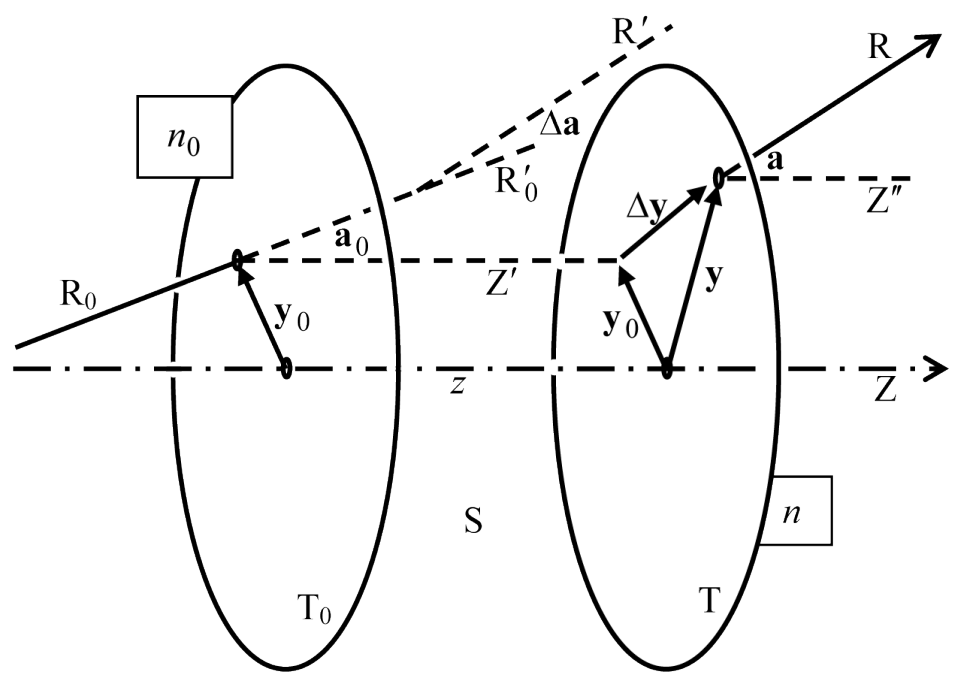

Figure 2 Deflection $\Delta \mathbf{a}$ (given by Equations 40 and 42) and transverse translation $\Delta \mathbf{y}$ (Equations 39 and 41) of a ray through an arbitrary system $\mathrm{S}$ in linear optics. This is a threedimensional generalization of Figure 1.

Instead of the $2 \times 2$ transference of Equation 1 we now have a $5 \times 5$ transference ${ }^{6}$ : 


$$
\mathbf{T}:=\left(\begin{array}{ccc}
\mathbf{A} & \mathbf{B} & \mathbf{e} \\
\mathbf{C} & \mathbf{D} & \boldsymbol{\pi} \\
\mathbf{o}^{\mathrm{T}} & \mathbf{0}^{\mathrm{T}} & 1
\end{array}\right) .
$$

The dilation, disjugacy, divergence and divarication now each become the $2 \times 2$ submatrices $\mathbf{A}, \mathbf{B}, \mathbf{C}$ and D. e and $\pi$ are each $2 \times 1$. They are the six fundamental properties of the system. $\mathbf{o}^{\mathrm{T}}$ is the matrix transpose of the $2 \times 1$ null matrix $\mathbf{0}$. The counterpart of Equation 2 is

$\mathbf{F}:=-\mathbf{C}$,

the dioptric power of the system.

The ray's incidence $(5 \times 1)$ is

$\boldsymbol{\gamma}_{0}:=\left(\begin{array}{c}\mathbf{y}_{0} \\ \boldsymbol{\alpha}_{0} \\ 1\end{array}\right)$

where $\mathbf{y}_{0}$ is the position and $\boldsymbol{\alpha}_{0}$ the reduced inclination of the ray relative to longitudinal axis Z. $\mathbf{y}_{0}$ and $\boldsymbol{\alpha}_{0}$ are $2 \times 1$, each consisting of Cartesian coordinates. $\boldsymbol{\alpha}_{0}$ is related to the ray's incident inclination $\mathbf{a}_{0}$ by

$$
\boldsymbol{\alpha}_{0}:=n_{0} \mathbf{a}_{0}
$$

where $n_{0}$ is the index of refraction before the system.

Similarly the ray's emergence is

$\boldsymbol{\gamma}:=\left(\begin{array}{l}\mathbf{y} \\ \boldsymbol{\alpha} \\ 1\end{array}\right)$.

where the reduced emergent inclination $\boldsymbol{\alpha}$ is related to the emergent inclination a by

$\boldsymbol{\alpha}:=n \mathbf{a}$.

The basic equation of linear optics is

$$
\mathbf{T} \boldsymbol{\gamma}_{0}=\boldsymbol{\gamma}
$$

Substituting from above one obtains the two matrix equations

$$
\mathbf{A y}_{0}+n_{0} \mathbf{B} \mathbf{a}_{0}+\mathbf{e}=\mathbf{y}
$$

and
$\mathbf{C y}_{0}+n_{0} \mathbf{D a}_{0}+\boldsymbol{\pi}=n \mathbf{a}$.

Solving Equation 30 for $\boldsymbol{\gamma}_{0}$ one obtains

$\boldsymbol{\gamma}_{0}=\mathbf{T}^{-1} \boldsymbol{\gamma}$

Because of symplecticity this is always possible. The inverse is

$\mathbf{T}^{-1}=\left(\begin{array}{ccc}\mathbf{D}^{\mathrm{T}} & -\mathbf{B}^{\mathrm{T}} & -\mathbf{D}^{\mathrm{T}} \mathbf{e}+\mathbf{B}^{\mathrm{T}} \boldsymbol{\pi} \\ -\mathbf{C}^{\mathrm{T}} & \mathbf{A}^{\mathrm{T}} & \mathbf{C}^{\mathrm{T}} \mathbf{e}-\mathbf{A}^{\mathrm{T}} \boldsymbol{\pi} \\ \mathbf{o}^{\mathrm{T}} & \mathbf{o}^{\mathrm{T}} & 1\end{array}\right)$

as is readily confirmed by direct evaluation of $\mathbf{T}^{-1} \mathbf{T}$ and $\mathbf{T T}^{-1}$. Then Equation 33 results in the pair of equations

$\mathbf{D}^{\mathrm{T}} \mathbf{y}-n \mathbf{B}^{\mathrm{T}} \mathbf{a}-\mathbf{D}^{\mathrm{T}} \mathbf{e}+\mathbf{B}^{\mathrm{T}} \boldsymbol{\pi}=\mathbf{y}_{0}$

and

$-\mathbf{C}^{\mathrm{T}} \mathbf{y}+n \mathbf{A}^{\mathrm{T}} \mathbf{a}+\mathbf{C}^{\mathrm{T}} \mathbf{e}-\mathbf{A}^{\mathrm{T}} \boldsymbol{\pi}=n_{0} \mathbf{a}_{0}$.

Corresponding to Equations 14 and 15 we define, for a ray, the transverse translation,

$\Delta \mathbf{y}:=\mathbf{y}-\mathbf{y}_{0}$.

and the deflection,

$\Delta \mathbf{a}:=\mathbf{a}-\mathbf{a}_{0}$.

Substituting from Equations 31 and 32 into Equations 37 and 38 we obtain the transverse translation and deflection of the ray in terms of its incidence:

$\Delta \mathbf{y}=(\mathbf{A}-\mathbf{I}) \mathbf{y}_{0}+n_{0} \mathbf{B} \mathbf{a}_{0}+\mathbf{e}$

and

$\Delta \mathbf{a}=\mathbf{C} \mathbf{y}_{0} / n+\left(n_{0} \mathbf{D} / n-\mathbf{I}\right) \mathbf{a}_{0}+\pi / n$.

Similarly, from Equations 35 to 38 , we obtain the transverse translation and deflection in terms of its emergence:

$\Delta \mathbf{y}=\left(\mathbf{I}-\mathbf{D}^{\mathrm{T}}\right) \mathbf{y}+n \mathbf{B}^{\mathrm{T}} \mathbf{a}-\mathbf{B}^{\mathrm{T}} \boldsymbol{\pi}+\mathbf{D}^{\mathrm{T}} \mathbf{e}$

and

$\Delta \mathbf{a}=\mathbf{C}^{\mathrm{T}} \mathbf{y} / n_{0}+\left(\mathrm{I}-n \mathbf{A}^{\mathrm{T}} / n_{0}\right) \mathbf{a}$

$+\left(\mathrm{A}^{\mathrm{T}} \boldsymbol{\pi}-\mathbf{C}^{\mathrm{T}} \mathbf{e}\right) / n_{0}$. 
Equations 39 and 40 are the generalized versions of Equations 16 and 17; they give the transverse translation and deflection of a ray in term of the ray's incidence. Equations 41 and 42 generalize Equations 18 and 19; they are in terms of the ray's emergence. Together Equations 39 to 42 represent the first objective of this note.

We now consider a few special cases.

If $\mathrm{Z}$ coincides with the optical axis of the system then $\mathbf{e}$ and $\pi$ are both null and these equations simplify accordingly.

If the indices of the media before and after the system are the same one obtains (from Equations 40 and 42) the generalizations of Equations 20 and 21:

$$
\Delta \mathbf{a}=\mathbf{C} \mathbf{y}_{0} / n+(\mathbf{D}-\mathbf{I}) \mathrm{a}_{0}+\pi / n \text {. }
$$

and

$$
\Delta \mathbf{a}=\mathbf{C}^{\mathrm{T}} \mathbf{y} / n_{0}+\left(\mathbf{I}-\mathbf{A}^{\mathrm{T}}\right) \mathbf{a}+\left(\mathbf{A}^{\mathrm{T}} \pi-\mathbf{C}^{\mathrm{T}} \mathbf{e}\right) / n_{0} .
$$

For a ray incident parallel to longitudinal axis $\mathrm{Z}$ $\mathbf{a}_{0}=\mathbf{o}$ and, hence, the transverse translation and deflection are

$$
\Delta \mathbf{y}=(\mathbf{A}-\mathbf{I}) \mathbf{y}_{0}+\mathbf{e}
$$

from Equation 39 and

$$
\Delta \mathbf{a}=\mathbf{C y}_{0} / n+\pi / n
$$

from Equation 40.

For thin systems $\mathbf{D}=\mathbf{I}$. If $\mathbf{y}_{0}$ is measured from the optical centre of a thin system in air then $\boldsymbol{\pi}=\mathbf{0}$ and Equation 43 reduces to Long's generalization ${ }^{7}$ of what is commonly called Prentice's equation but probably better called Imbert's equation ${ }^{8,9}$.

\section{Nodal rays}

A nodal ray through a system is one which is not deflected, that is, $\Delta \mathbf{a}=\mathbf{0}$ or, equivalently ${ }^{3}$

$$
\mathbf{a}_{0}=\mathbf{a} \text {. }
$$

Hence, from Equation 40 a nodal ray satisfies

$$
\mathbf{C} \mathbf{y}_{0}+\left(n_{0} \mathbf{D}-n \mathbf{I}\right) \mathbf{a}_{0}+\pi=\mathbf{o} \text {. }
$$

This can be solved to give

$$
\mathbf{y}_{0}=\mathbf{C}^{-1}\left(n \mathbf{I}-n_{0} \mathbf{D}\right) \mathbf{a}-\mathbf{C}^{-1} \boldsymbol{\pi},
$$

provided $\mathbf{C}$ is nonsingular, and

$$
\mathbf{a}=\left(n \mathbf{I}-n_{0} \mathbf{D}\right)^{-1}\left(\mathbf{C y}_{0}+\pi\right)
$$

provided $n \mathbf{I}-n_{0} \mathbf{D}$ is nonsingular. In most cases of interest these matrices are nonsingular. When they are singular there may be either no solution or an infinity of solutions, exceptional cases that are not examined here. The coefficient of a in Equation 49 is the negative of the incident nodal characteristic defined elsewhere. ${ }^{3}$

Equation 49 gives the incident position of a nodal ray with inclination a and Equation 50 the inclination of the nodal ray incident at $\mathbf{y}_{0}$. Corresponding equations can be obtained in terms of the emergent position $\mathbf{y}$ from Equation 42.

\section{Optical axis}

By definition ${ }^{2}$ an optical axis is a straight line through the system along which a ray both enters and leaves the system. Thus $\Delta \mathbf{a}=\mathbf{o}$ and the optical axis is a nodal ray. Hence Equation 48 holds. Also

$\Delta \mathbf{y}=z \mathbf{a}$

where $z$ is the length of the system. Thus, from Equation 39 , one finds that

$z \mathbf{a}=(\mathbf{A}-\mathbf{I}) \mathbf{y}_{0}+n_{0} \mathbf{B a}+\mathbf{e}$.

Equations 48 and 52 can be written

$$
(\mathbf{A}-\mathbf{I}) \mathbf{y}_{0}+\left(n_{0} \mathbf{B}-z \mathbf{I}\right) \mathbf{a}=-\mathbf{e}
$$

and

$\mathbf{C} \mathbf{y}_{0}+\left(n_{0} \mathbf{D}-n \mathbf{I}\right) \mathbf{a}=-\pi$.

These two equations can be combined as the single matrix equation

$$
\left(\begin{array}{cc}
\mathbf{A}-\mathbf{I} & n_{0} \mathbf{B}-z \mathbf{I} \\
\mathbf{C} & n_{0} \mathbf{D}-n \mathbf{I}
\end{array}\right)\left(\begin{array}{c}
\mathbf{y}_{0} \\
\mathbf{a}
\end{array}\right)=-\left(\begin{array}{l}
\mathbf{e} \\
\boldsymbol{\pi}
\end{array}\right)
$$

obtained before ${ }^{2}$ by a different method. As before we put

$$
\mathbf{P}:=\left(\begin{array}{cc}
\mathbf{A}-\mathbf{I} & n_{0} \mathbf{B}-z \mathbf{I} \\
\mathbf{C} & n_{0} \mathbf{D}-n \mathbf{I}
\end{array}\right) .
$$

Also putting 


$$
\mathbf{d}:=\left(\begin{array}{c}
\mathbf{y}_{0} \\
\mathbf{a}
\end{array}\right)
$$

and

$$
\delta:=\left(\begin{array}{l}
\mathrm{e} \\
\pi
\end{array}\right)
$$

we rewrite Equation 55 as

$$
\mathbf{P d}=-\delta \text {. }
$$

Provided $\mathrm{P}$ is nonsingular

$$
\mathbf{d}=-\mathbf{P}^{-1} \delta
$$

the result obtained before ${ }^{2}$ but by a simpler method. For most systems of interest $\mathbf{P}$ is nonsingular. ${ }^{5} \mathbf{d}$ (Equation 57) gives the position $\mathbf{y}_{0}$ of the optical axis in the entrance plane $T_{0}$ of the system and $\mathbf{a}$ the inclination of the optical axis.

\section{Concluding remarks}

Given the transference of an optical system Equations 40 and 42 allow one to calculate the deflection of a ray traversing the system; Equation 40 gives the deflection in terms of the position and inclination of the ray at incidence and Equation 42 gives in terms of the position and inclination at emergence. The equations are general and, within the limitations of linear optics, hold for all optical systems whether or not they contain astigmatic and relatively decentred refracting elements. The same holds with respect to transverse translation and Equations 39 and 41.

Equations 43 to 46 specialize these results for particular situations.

Equations governing nodal rays (Equations 48 to 50) arise naturally from the analysis because nodal rays are not deflected.

Equations governing the optical axis of a system (Equations 59 and 60) also arise naturally, and more simply than before ${ }^{2}$, because an optical axis is that particular nodal ray which enters and leaves the system on the same straight line.

\section{Acknowledgement}

Support from the National Research Foundation to WF Harris is gratefully acknowledged.

\section{References}

1. Mathebula SD. Quantitative Analysis of the Linear Optical Character of the Anterior Segment of the Eye. DPhil dissertation, University of Johannesburg, 2009 submitted.

2. Harris WF. Optical axes of eyes and other optical systems. Optom Vis Sci 200986 537-541.

3. Harris WF. Nodes and nodal points and lines in eyes and other optical systems. Ophthal Physiol Opt 201030 2442.

4. Guillemin V, Sternberg S. Symplectic Techniques in Physics. Cambridge University Press, Cambridge, 1984.

5. Harris WF. Dioptric power: its nature and its representation in three- and four-dimensional space. Optom Vis Sci 1997 74 349-366.

6. Harris WF. Paraxial ray tracing through noncoaxial astigmatic optical systems, and a augmented system matrix. $O p$ tom Vis Sci 199471 282-285.

7. Long WF. A matrix formalism for decentration problems. Am J Optom Physiol Opt 197653 27-33.

8. Bennett AG. Prismatic effects of spherical and astigmatic lenses: Imbert's pioneer analysis of 1886. Ophthal Physiol Opt 199010 397-398.

9. Harris WF. Effect of spectacle and contact lenses on the effective corneal refractive zone. Clin Exper Optom 2009 92 99-103. 